\title{
A comparative study of danazol and norethisterone in dysfunctional uterine bleeding presenting as menorrhagia
}

\author{
M. Bonduelle*, J.J. Walker and A.A. Calder ${ }^{\dagger}$ \\ Department of Obstetrics and Gynaecology, Glasgow Royal Infirmary, Glasgow G21 3UW, UK
}

\begin{abstract}
Summary: This randomized open study compared the efficacy and safety of norethisterone, $5 \mathrm{mg}$ three times a day from day 19 to 26 , and danazol, $200 \mathrm{mg}$ daily, in the treatment of dysfunctional uterine bleeding presenting as menorrhagia. Clinical criteria were employed to confirm the diagnosis, and subjective assessment of the condition was performed during one pre-treatment and three treatment cycles. Fourteen patients commenced norethisterone and 10 danazol.

Bleeding intensity scores were significantly lower with danazol than with norethisterone, and patients assessed their blood loss to be significantly less with danazol than with norethisterone. Associated symptoms of backache and abdominal pain were improved to a similar degree by both treatments. Adverse reactions were reported with similar frequency and were of a similar nature in both treatment groups.
\end{abstract}

\section{Introduction}

Menorrhagia is a common complaint resulting in annual general practitioner consultation rates of 20.4 per 1,000 women. ${ }^{1}$ In those patients in whom no underlying pathology is apparent and menorrhagia is attributable to dysfunctional uterine bleeding (often termed primary menorrhagia), it has been reported that as many as $62 \%$ would be found by objective measurement to have a mean menstrual loss within the normal range. ${ }^{2}$ Since the majority of gynaecology clinics do not have facilities to assess menorrhagia objectively, a trial of medical treatment prior to surgical intervention such as hysterectomy, with its attendant morbidity and expense, is generally appropriate.

Various medical regimes have been used in the treatment of primary menorrhagia. Progestational agents, most commonly norethisterone, are widely employed for this complaint, despite the lack of published evidence that they are effective, since they are considered to induce secretory changes in the endometrium, preventing endometrial hyperplasia and regulating the menstrual cycle. Danazol, a synthetic steroid, in contrast, has been shown to

Correspondence: M. Bonduelle, M.D., M.R.C.O.G.

*Present address: University Department of Obstetrics and Gynaecology, Harare, Zimbabwe

${ }^{\dagger}$ Present address: University Department of Obstetrics and Gynaecology Centre for Reproductive Endocrinology, 37 Chalmers Street, Edinburgh EH3 9EW

Accepted: 25 March 1991 be an effective treatment for primary menorrhagia, substantially reducing menstrual blood $\operatorname{loss}^{3}$ as well as its perceived intensity. ${ }^{4}$ Danazol acts at the pituitary, ${ }^{5}$ ovarian $^{6}$ and endometrial level ${ }^{7}$ and induces endometrial atrophy. ${ }^{8}$

In the absence of previously published comparisons, a pilot study was undertaken to compare the efficacy, tolerability and safety of danazol and norethisterone in treating this condition.

\section{Materials and methods}

\section{Study design}

Thirty patients diagnosed as having dysfunctional uterine bleeding presenting as menorrhagia (primary menorrhagia) were recruited from the Menstrual Disorders Clinic at the Royal Infirmary, Glasgow. Selection was based on clinical criteria: complaint of menstrual loss requiring more than 5 pads/tampons per day for longer than 6 days cycle; presence of flooding or clots on any day of the cycle; presence of secondary anaemia; excessive menstrual loss proving socially and domestically disruptive. Underlying pathology was excluded by means of history, examination and dilatation and curettage within the preceding 3 to 12 months.

All patients provided written, informed consent prior to commencing the study, which had hospital ethics committee approval.

Initial screening included menstrual, obstetric and general medical histories, general and vaginal 
examinations together with haematological and liver function tests.

Patients were reviewed after 1 cycle without treatment having maintained a daily record of the duration and heaviness of menstrual loss, use of sanitary material and intensity of any abdominal pain or backache. Reports relating to possible adverse events were also noted and laboratory tests repeated if abnormal. If the diagnosis of primary menorrhagia was confirmed, the patient was recruited into the study and randomly assigned to three cycles of treatment with either danazol, $200 \mathrm{mg}$ daily, or norethisterone, $5 \mathrm{mg}$ three times a day from day 19 to 26 .

Daily diaries were required throughout treatment and reviewed after each menstrual period, as above. Heaviness of bleeding was rated on a descriptive scale, and scored for each day of bleeding from 1 to 7 (where $1=$ spotting and $7=$ flooding). Daily scores were combined to yield a bleeding intensity score for each menstrual period. Scores for abdominal pain and backache were derived similarly from a descriptive scale, scored from 1 to 3 (where $1=$ mild and $3=$ severe).

At each hospital visit the patients' assessment of their condition in relation to baseline (greatly improved to much worse), the efficacy of treatment (completely effective to ineffective), the severity of blood loss (spotting to flooding) and dysmenorrhoea (absent to severe), during the most recent menstrual period were recorded.

\section{Statistical analysis}

Comparisons between the two treatment groups were made using the Wilcoxon two sample test, and those within, using the Wilcoxon matched pairs test. Results relating to the first menstrual loss after the start of treatment were not used for comparison since the two groups were not at a comparable stage of treatment. Danazol patients had commenced treatment only at the start of that period whereas those taking norethisterone had completed one cycle of treatment by this stage.

\section{Results}

\section{Study population}

Thirty patients were recruited into the study; of these 15 were randomized to receive danazol and 15 norethisterone. Six patients were excluded from the analysis ( 5 from the danazol group, 1 from the norethisterone group), 4 because they did not return and, hence provided no data, following randomization, and 2 because selection criteria had not been met ( 1 - Wolf Parkinson White syndrome; 1 - epileptic).
There were no significant differences between the two treatment groups in age, number of pregnan- $\frac{3}{\alpha}$ cies, duration of menorrhagia or menstrual history $\stackrel{\mathbb{Q}}{c}$ (Table I).

The variables used to assess efficacy were also comparable at baseline except for abdominal pain $\underset{\nabla}{0}$ score, which was higher in those receiving danazol (Table II).

\section{Withdrawals}

Eight patients withdrew during the course of the $\overrightarrow{0}$ study, 4 from each treatment group. All data for these patients were included in the analyses up to $\vec{\omega}$ the point of withdrawal. Of those withdrawing $\frac{D}{0}$ during danazol treatment, 2 did so because of $\frac{0}{3}$ events thought to be related to medication (allergic rash;. weight gain, hypertension, musculoskeletal .

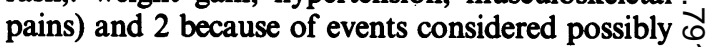
related to medication (vomiting; mood changes with $\vec{\phi}$ suicidal thoughts). In the norethisterone group, one $\mathscr{W}_{\tilde{W}}$ patient withdrew because of events related to 0 medication (headaches) and three as a consequence of events possibly related to medication (depression, $\bar{c}$ light headedness, sickness; musculoskeletal pain; $\frac{\mathbb{D}}{0}$ mood changes, lack of concentration).

\section{Efficacy}

Daily assessments (Table II) Bleeding intensit scores were significantly lower with danazol tha with norethisterone for the third menses. This score was also significantly improved with danazol, but not with northisterone, by the 2nd and 3rd menses in comparison with baseline $(P<0.02)$ as were the number of pads/tampons used $(P<0.05)$. Some reduction in the symptoms of backache and abdominal pain accompanied both treatments although between treatment comparisons were not significant.

Clinic assessments The patients' overall assessments of their condition and the efficacy of treatment demonstrated no significant differences

Table I Admission characteristics

\begin{tabular}{|c|c|c|c|c|}
\hline & $\begin{array}{l}\text { Dan } \\
(n=\end{array}$ & $\begin{array}{l}\text { azol } \\
10)\end{array}$ & $\begin{array}{c}\text { Norethis } \\
\quad(n=\end{array}$ & $\begin{array}{l}\text { terone } \\
14)\end{array}$ \\
\hline & Mean & s.d. & Mean & s.d. \\
\hline Age (yrs) & 36.1 & 5.9 & 39.2 & 5.3 \\
\hline No. live births & 2.1 & 1.0 & 2.9 & 1.1 \\
\hline No. of terminations & 0.1 & 0.3 & 0.4 & 0.8 \\
\hline Duration menorrhagia (yrs) & 4.8 & 5.3 & 3.8 & 5.2 \\
\hline Length of cycle (days) & 26.2 & 3.3 & 28.0 & 4.6 \\
\hline Days of bleeding & 7.1 & 1.9 & 7.9 & 2.5 \\
\hline Average no. pads/cycle & 32.2 & 15.6 & 34.4 & 15.7 \\
\hline Average no. tampons/cycle & 7.4 & 15.7 & 7.4 & 16.7 \\
\hline
\end{tabular}


Table II Daily assessments (mean \pm s.d.)

\begin{tabular}{|c|c|c|c|c|c|c|}
\hline & $\begin{array}{c}\text { Baseline } \\
(n=10)\end{array}$ & $\begin{array}{c}\text { Danazol } \\
\text { 2nd menses } \\
(n=7)\end{array}$ & $\begin{array}{c}\text { 3rd menses } \\
\quad(n=6)\end{array}$ & $\begin{array}{l}\text { Baseline } \\
(n=14)\end{array}$ & $\begin{array}{l}\text { Norethisterone } \\
2 \text { 2nd menses } \\
(n=11)\end{array}$ & $\begin{array}{c}3 r d \text { menses } \\
(n=10)\end{array}$ \\
\hline Bleeding intensity score & $31.9 \pm 12.4$ & $14.1 \pm 11.3^{\ddagger}$ & $15.0 \pm 9.4^{* \pm}$ & $32.9 \pm 15.2$ & $23.7 \pm 6.5$ & $23.6 \pm 9.6$ \\
\hline No. of pads/tampons used & $46.9 \pm 37.2$ & $13.4 \pm 12.2^{\dagger}$ & $14.0 \pm 12.2^{\dagger}$ & $37.6 \pm 27.9$ & $23.4 \pm 11.6$ & $25.1 \pm 12.6$ \\
\hline No. days bleeding & $7.3 \pm 2.7$ & $4.3 \pm 2.6$ & $5.2 \pm 2.6$ & $7.6 \pm 3.3$ & $5.8 \pm 1.5$ & $5.8 \pm 2.2$ \\
\hline No. days backache & $4.8 \pm 2.6$ & $2.4 \pm 3.1$ & $3.7 \pm 3.4$ & $4.6 \pm 2.5$ & $3.2 \pm 2.4$ & $2.4 \pm 2.8^{\ddagger}$ \\
\hline Backache score & $10.9 \pm 7.2$ & $4.7 \pm 6.1^{\ddagger}$ & $6.5 \pm 7.1$ & $10.5 \pm 6.2$ & $5.7 \pm 6.8$ & $5.0 \pm 7.0^{\dagger}$ \\
\hline No. days abdominal pain & $4.8 \pm 2.8$ & $2.9 \pm 2.9$ & $1.8 \pm 2.2^{\dagger}$ & $2.8 \pm 2.2$ & $2.8 \pm 1.8$ & $1.9 \pm 2.5$ \\
\hline Abdominal pain score & $10.3 \pm 5.9^{*}$ & $5.7 \pm 6.2$ & $3.0 \pm 4.0$ & $5.6 \pm 4.6$ & $5.4 \pm 3.0$ & $4.2 \pm 6.7$ \\
\hline
\end{tabular}

${ }^{*} P<0.05$ vs norethisterone; ${ }^{\dagger} P<0.05$ vs baseline; ${ }^{\ddagger} P<0.02$ vs baseline.

between the two treatments. However, by the second visit during treatment the patients assessed their blood loss to be significantly less with danazol than with norethisterone (none to moderate, 5 of 7 with danazol vs 3 of 10 with norethisterone, $P<0.02)$. Dysmenorrhoea improved to a significant degree with norethisterone $(P<0.05)$ but not with danazol, although this may reflect the small numbers available for analysis.

\section{Safety}

Minor adverse reactions were common and reported with similar frequency in both groups (Table III). Two patients reported voice changes during norethisterone which resolved either during treatment or after discontinuation of treatment. Although the cause of these voice changes is unknown, it is possible that a concurrent upper respiratory-tract infection was responsible.

Although weight gain was complained of by 8 patients on danazol and 7 on norethisterone, during treatment, only 4 patients recorded an increase of greater than $3 \mathrm{~kg}$ ( 3 on danazol, 1 on

Table III Patient reported adverse reactions to treatment

\begin{tabular}{lcc}
\hline & \multicolumn{2}{c}{ No. of events } \\
& Danazol & Norethisterone \\
\hline Voice changes & 0 & 2 \\
Loss concentration/depression/ & 5 & 7 \\
$\quad$ tiredness/irritability/strange & & \\
$\quad$ feelings & & \\
Headache & 4 & 1 \\
Skin changes/rashes & 6 & 3 \\
Gastro-intestinal symptoms & 7 & 5 \\
Weight gain & 8 & 7 \\
Bloatedness & 6 & 3 \\
Total no. patients & 10 & 14 \\
$\quad$ reporting adverse reactions & & \\
\hline
\end{tabular}

norethisterone). Three patients were recorded as having diastolic blood pressures $>90 \mathrm{mmHg}$, in association with systolic pressures $>140 \mathrm{mmHg}$ for the first time during treatment with danazol, although this resulted in withdrawal from the study in only one patient. However, since the conditions under which blood pressure was measured were not standardized these findings are of doubtful significance.

There were no clinically significant changes in blood count, or liver function tests associated with either treatment, nor were there any clinically serious adverse reactions during the study.

There were no unexpected side effects seen in patients treated with danazol. However, norethisterone was associated with a higher than expected incidence of side effects not widely documented during such treatment.

\section{Discussion}

Primary menorrhagia is normally managed symptomatically, and although patients are poor at assessing absolute blood loss, they are more able to assess change. ${ }^{2}$ In keeping with ordinary clinical practice, then, this pilot study employed subjective assessments of the principal variables.

Danazol was employed at a dose of $200 \mathrm{mg}$ daily, a regime which has been demonstrated previously $y^{3,4}$ to provide effective treatment for primary menorrhagia. The regimen used for norethisterone therapy is that recommended by the manufacturers. since no objective data were available to indicate the most suitable dose.

Whilst conclusions from this study are limited by the small number of subjects, the findings confirm the clinical efficacy of danazol in controlling menorrhagia, significant improvements being seen in both the prospective daily and clinic assessments of this symptom. The findings also suggest that danazol is more effective than norethisterone, the efficacy of which, in controlling menorrhagia, 
remains in doubt, although it may, like danazol, benefit associated symptoms. Poorer compliance associated with the three times a day norethisterone regimen in comparison with the once daily danazol regimen may have contributed to the differences in efficacy demonstrated in this study.

The high level of adverse events reported probably reflects the particular attention given to this aspect of the study in the baseline as well as the treatment phase. None was clinically serious, but it is notable that the spectrum of complaints and also the frequency of discontinuation of treatment, was

\section{References}

1. Rees, M. Menorrhagia. $\mathrm{Br}$ Med J 1987, 294: 759-762.

2. Fraser, I.S., McCarron, G. \& Markham, R. A preliminary study of factors influencing perception of menstrual blood loss volume. Am J Obstet Gynecol 1987, 149: 788-793.

3. Chimbira, T.H., Anderson, A.B.M., Naish, C., Cope, E. \& Turnbull, A.C. Reductions of menstrual blood loss by danazol in unexplained menorrhagia: lack of effect of placebo. $\mathrm{Br} J$ Obstet Gynaecol 1980, 87: 1152-1158.

4. Lamb, M.P. Danazol in menorrhagia: a double blind placebo controlled trial. J Obstet Gynecol 1987, 7: 212-216.

5. Chimbira, T.H. Effect of danazol on serum gonadotrophins and steroid hormone concentrations in women with menorrhagia. Br J Obstet Gynaecol 1980, 87: 330-336. comparable for the two medications.

Since this study was undertaken, a report of objective measurement of blood loss in small groups of patients ${ }^{9}$ casts further doubt on the efficacy of norethisterone, although it confirms that of danazol. Since norethisterone is very widely used, a detailed assessment of its efficacy and safety in comparison to danazol is overdue. Should such a study confirm the findings discussed here then danazol could usefully be employed as first line therapy in the management of dysfunctional uterine bleeding presenting as menorrhagia.

6. Steingold, K.A. Danazol inhibits steroidogenesis by the human ovary in vivo. Fertil Steril 1986, 45: 649-654.

7. Kauppila, A. Cystosol oestrogen and progestin receptor concentrations and 17 $\beta$-hydroxysteroid dehydrogenase activities in the endometrium and endometriotic tissue. Acta Obstet Gynecol Scand 1984, (Suppl. 123): 45-49.

8. Jeppsson, S. et al. Short-term effects of danazol on endometrial histology. Acta Obstet Gynecol Scand 1984, (Suppl. 123): 41-44.

9. Cameron, I.T. Dysfunctional uterine bleeding. Baillières Clin Obstet Gynaecol 1989, 3: 315-327. 